\title{
Anfione-Niobe e Zeto-Aedon: la fondazione di Tebe nel dramma attico
}

Amphion-Niobé et Zéthos-Aédon : la fondation de Thèbes dans le drame attique Amphion-Niobe and Zethos-Aëdon: The Foundation of Thebes in Attic Drama

\section{Sabrina Mancuso}

\section{CpenEdition}

\section{Journals}

\section{Edizione digitale}

URL: https://journals.openedition.org/gaia/357

DOI: $10.4000 /$ gaia.357

ISSN: $2275-4776$

Editore

UGA Éditions/Université Grenoble Alpes

Edizione cartacea

ISBN: 978-2-37747-057-0

ISSN: $1287-3349$

\section{Notizia bibliografica digitale}

Sabrina Mancuso, «Anfione-Niobe e Zeto-Aedon: la fondazione di Tebe nel dramma attico», Gaia [Online], 21 | 2018, online dal 01 novembre 2018, consultato il 09 décembre 2021. URL: http:// journals.openedition.org/gaia/357 ; DOI: https://doi.org/10.4000/gaia.357

Questo documento è stato generato automaticamente il 9 décembre 2021.

Gaia. Revue interdisciplinaire sur la Grèce archaïque 


\section{Anfione-Niobe e Zeto-Aedon: la fondazione di Tebe nel dramma attico}

Amphion-Niobé et Zéthos-Aédon : la fondation de Thèbes dans le drame attique Amphion-Niobe and Zethos-Aëdon: The Foundation of Thebes in Attic Drama

\section{Sabrina Mancuso}

\section{Introduzione}

\subsection{Problem setting e metodologia}

1 Il presente contributo si propone di far luce sulla ricezione di alcuni miti arcaici del ciclo tebano - in particolare quello relativo a Zeto e alla sua sposa Aedon - nell'età classica, tramite un'analisi dei loro adattamenti e riletture. L'obiettivo principale della ricerca proposta consiste nell'indagare come il mito di Aedon si trasformi, nel momento in cui viene introdotto nel dramma attico, e come tale trasformazione sia imputabile allo spostamento dell'ambientazione del mito da Tebe in Tracia, dovuto a motivazioni politiche. La seconda parte del presente contributo si propone di offrire un quadro completo dei miti di Anfione e Zeto e delle loro spose nell'età arcaica, in modo da delineare il contesto storico e letterario, in cui questi miti si inquadravano prima dell'età classica. L'ultimo paragrafo di questa sezione è dedicato all'indagine dei significati metapoetici del canto di Anfione, i quali trovano un parallelo nel canto di Aedon.

2 Da un punto di vista metodologico, mi propongo di distinguermi dagli studi precedenti, analizzando il primo campo d'indagine prospettato attraverso un confronto sistematico - talvolta coadiuvato da una comparazione intertestuale - fra le fonti arcaiche del mito dell'usignolo e i riferimenti ad esso presenti in tragedia. Inoltre, mi propongo di arricchire quest'analisi, presentando un breve studio relativo alle etimologie popolari dei protagonisti maschili delle due versioni di questo mito, utile all'interpretazione 
letteraria della rilettura che ne viene effettuata nel dramma attico. La seconda sezione sarà invece caratterizzata da un confronto fra le fonti del mito di Anfione e Zeto $\mathrm{e}$ quelle delle saghe asiatiche. Il campo di indagine relativo ai significati metapoetici del mito di Anfione e di quello dell'usignolo sarà infine analizzato attraverso un commento generale ai testi, arricchito da un confronto con paralleli letterari.

\subsection{Tebe nell'epos arcaico}

3 Secondo il ciclo tebano, il fondatore di Tebe è generalmente Cadmo ([Apollodoro], Biblioteca, III, 1,4). È, dunque, osservabile una narrativa di fondazione relativamente standard: un enigma causa il ricorso a un oracolo, e un eroe, Cadmo, viaggia da un'altra terra per fondare la città ${ }^{1}$ Ma, in aggiunta alla storia di Cadmo, c'è anche la storia dei gemelli Anfione e Zeto, eroi locali tebani. In Omero, Cadmo è menzionato solo una volta, e come padre di Ino, non come fondatore di Tebe. Anfione e Zeto sono, invece, indicati come fondatori di Tebe ${ }^{2}$ :

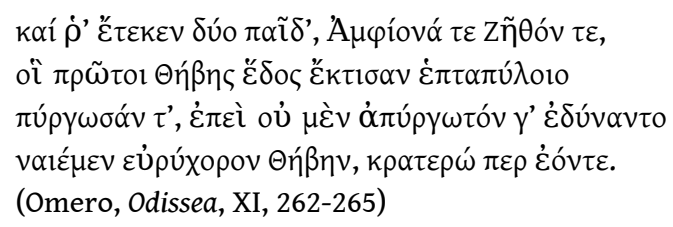

4 Nei poemi omerici, Anfione e Zeto sono i gemelli figli di Zeus e Antiope, figlia di Asopo (Omero, Odissea, XI, 260-265) ${ }^{3}$. Entrambi presero parte alla spedizione degli Argonauti e costruirono le mura di Tebe, di cui divennero signori (Omero, Odissea, XI, 260-265). Zeto sposò Aedon ${ }^{4}$, con cui generò Itilo ma, dopo l'uccisione di quest'ultimo effettuata dalla madre, morì di preoccupazione e fu sepolto a Tebe ${ }^{5}$ :

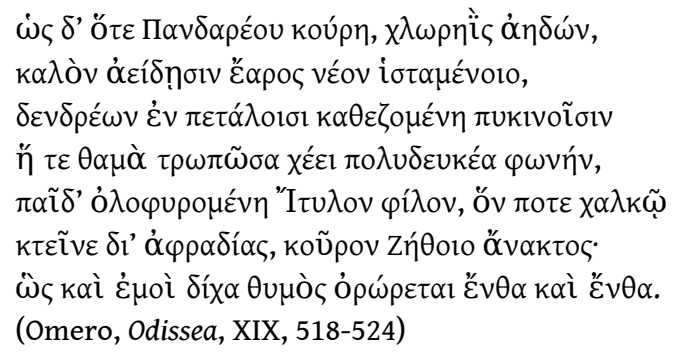

5 Secondo Esiodo (fr. 183 M.-W.), Anfione sposò invece Niobe, figlia di Tantalo. Conformemente alla testimonianza di Ferecide (III, fr. 124 Jacoby) ${ }^{6}$, i gemelli furono spesso messi in parallelo con Castore e Polluce. Ferecide (III, fr. 124 Jacoby) riferisce, inoltre, una tradizione, in base alla quale Zeto e Aedon avevano anche un secondo figlio, chiamato Neide ${ }^{7}$. Secondo fonti più tarde, i gemelli, esposti dopo la nascita per volontà di Lico e Dirce, gli zii di Antiope che la tenevano prigioniera, furono allevati da pastori. Quando ad Antiope riuscì di sfuggire alla prigionia per raggiungere i propri figli, i gemelli vendicarono le sofferenze della madre uccidendo Dirce. La storia di concepimento, nascita e riabilitazione finale di Anfione e Zeto fu sviluppata, se non in parte inventata, da Euripide nella sua Antiope ${ }^{8}$. Nella letteratura successiva, i gemelli furono spesso presentati come esempio del contrasto fra lo stile di vita pratico e quello filosofico (Platone, Gorgia, $485 \mathrm{e}, 506 \mathrm{~b}$; Rhetorica ad Herennium, II, 43; Cicerone, De oratore, II, 155) 9 . Piuttosto chiaramente, Apollonio Rodio (I, 738-740) mostrò la loro essenza basilarmente diversa: secondo lui, il robusto pastore Zeto muoveva le pietre con la forza muscolare, mentre al suono della lira del musico Anfione queste lo seguivano da sé. Dopo la morte dei suoi figli, Anfione attaccò il tempio di Apollo e fu, 
per questo, ucciso dalle sue frecce (Igino, Fabulae, IX). Secondo altre fonti, invece, si suicidò (Ovidio, Metamorfosi, VI, 271-272) o divenne pazzo (Luciano, De saltatione, XLI). Il matrimonio di Anfione con Niobe potrebbe rappresentare l'unione di due elementi della popolazione di base; non a caso, l'origine di Niobe può essere stabilita in Lidia.

\section{Il mito dell'usignolo da Omero alla tragedia}

\subsection{L'usignolo come sposa di Zeto: la versione arcaica del mito}

6 Al mito di Anfione e Zeto sono strettamente connessi i miti riguardanti le loro spose Niobe e Aedon, entrambe riconducibili ad origini asiatiche e menzionate nei poemi omerici.

7 Niobe era sposata ad Anfione, con cui generò molti figli ${ }^{10}$. Si vantò, dunque, di esser più feconda di Leto che, sdegnata, le scatenò contro Apollo e Artemide, i quali fecero strage dell'intera prole. Allora, Niobe andò dal padre Tantalo al Sipilo e lì, dopo aver pregato Zeus, fu trasformata in pietra ${ }^{11}$.

È possibile ricostruire la versione arcaica del mito di Aedon attraverso tre fonti, che si differenziano reciprocamente per alcuni particolari: il già citato Omero (Odissea, XIX, 518-524), Ferecide ${ }^{12}$ e lo scolio B2 ${ }^{13}$.

A. Omero allude al mito, ricordando come l'usignolo Aedon, che in origine era una donna nata da Pandareo, pianga per aver ucciso il proprio figlio Itilo, avuto da Zeto.

B. Secondo la tradizione riportata da Ferecide, Aedon, invidiosa dell'abbondante prole della cognata Niobe, decide di ucciderne il figlio maggiore, Amaleo. Per errore, Aedon uccide però il proprio figlio Itilo, che dormiva con Amaleo. Questo è il nucleo essenziale del mito, generalmente noto come versione tebana del mito di Aedon.

C. Lo scolio B2 contiene un ulteriore dettaglio, non menzionato dalle altre fonti: Aedon, dopo aver assassinato Itilo ma prima di essere trasformata in usignolo, è inseguita da Zeto.

\subsection{L'usignolo come sposa di Tereo: $\mathrm{i}$ significati politici assunti dal mito in tragedia}

9 La versione tragica del mito, deducibile da alcuni riferimenti presenti nei testi dei tre tragici ${ }^{14}$ e dai frammenti del Tereo di Sofocle, presenta numerosi cambiamenti. Conformemente ad essa ${ }^{15}$, Procne, figlia del re di Atene Pandione, sposa il re di Tracia Tereo e gli dà un figlio, Iti. Successivamente, Procne chiede a Tereo di recarsi ad Atene, per portarle la sorella Filomela. Tereo accetta, ma stupra Filomela sulla via del ritorno e le taglia la lingua, affinché nessuno conosca il suo gesto. Filomela riesce a comunicare con Procne, ricamando su un tessuto una narrazione dell'affronto subito. Le due sorelle, per vendetta, uccidono e fanno a pezzi Iti, per poi imbandirne le carni a Tereo. Resosi conto di ciò che ha mangiato, Tereo insegue le donne, con intenzioni omicide. A quel punto, Procne e Filomela vengono tramutate dagli dei rispettivamente in usignolo e rondine; Tereo, invece, si trasforma in un'upupa.

Nella versione tragica del mito, il nuovo protagonista maschile Tereo è, al contrario dell'irreprensibile Zeto, un violento stupratore. Mentre il tebano Zeto non ebbe alcuna colpa nell'uccisione del fanciullo, il trace Tereo è la ragione principale di questo delitto, 
in quanto scatenò la vendetta della sua sposa col proprio comportamento, caratterizzato da infedeltà e violenza.

11 Il Tereo è il dramma che presenta i più significativi adattamenti programmatici del mito dell'usignolo. L'uso di questo mito in chiave politica, da parte di Sofocle, è evidente dal fatto che Tucidide avverte la necessità di difendere il re di Tracia Tere dall'accusa di essere imparentato con Tereo, adducendo come motivazione delle sue argomentazioni il fatto che Tereo - a differenza di Tere - era un trace di Daulia ${ }^{16}$. Tere apparteneva alla tribù degli Odrisi; a lui succedette il figlio Sitalce, il quale intraprese una politica filo-ateniese, che culminò in un trattato di pace stipulato nel 431. Tale trattato fu, però, violato dallo stesso Sitalce, che passò dalla parte dei Macedoni. Atene era, perciò, intorno al 431, divisa fra tendenze filo-tracie e anti-tracie; nel 429 essa era, invece, verosimilmente dominata dall'ostilità nei confronti dei Traci. Il bersaglio polemico di Tucidide potrebbe essere, dunque, Sofocle, colui che - probabilmente per primo ambientò il mito dell'usignolo in Tracia ${ }^{17}$. Tale ambientazione implica conseguentemente una certa ostilità nei confronti dei Traci.

Un altro elemento del mito, che probabilmente è da considerarsi innovazione di Sofocle, è la glossotomia subita da Filomela. Tale innovazione ha, verosimilmente, due finalità: caratterizzare il barbaro Tereo in modo violento e rendere necessaria l'introduzione di un messaggio scritto, fondamentale al riconoscimento delle due sorelle ${ }^{18}$. Non a caso, la glossotomia e il messaggio scritto sono elementi carichi di implicazioni metaforiche: essi tematizzano lo scontro fra la civiltà - la quale è caratterizzata dall'alfabetizzazione - e la barbarie - contrassegnata dall'ostilità nei confronti della scrittura. La glossotomia di Filomela effettuata da Tereo allude chiaramente all'ionүopí $\alpha$, osteggiata dai barbari; tale azione viene successivamente punita proprio da un atto di scrittura, appunto dalla "voce della spola» ${ }^{19}$. La "voce della spola» si carica, dunque, di due valori simbolici: l'ínүopía - tipica della civiltà ateniese - e l'abilità nella tessitura tipica delle donne ateniesi; tali elementi porteranno le principesse di Atene a sconfiggere il barbaro Tereo ${ }^{20}$. In questo modo, viene dimostrata la superiorità ateniese sui barbari, superiorità evidente tanto sul piano pratico quanto su quello artistico, sfere cui afferiscono sia la scrittura sia la tessitura.

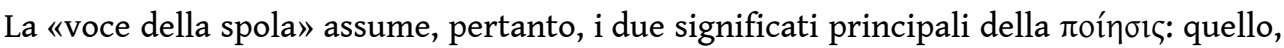
cioè, di creazione dal nulla ${ }^{21}$ e quello di creazione poetica ${ }^{22}$. Un'altra probabile innovazione sofoclea che allude al carattere barbarico di Tereo è la metamorfosi in upupa, la quale compare per la prima volta nel Tereo:

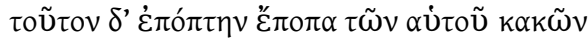

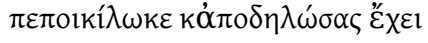

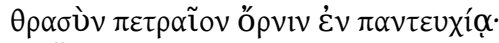

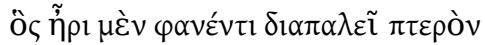

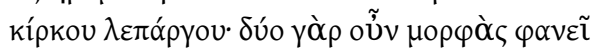

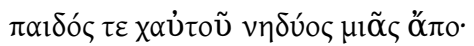

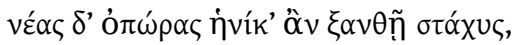

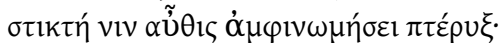

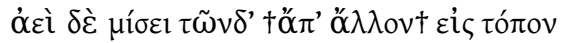

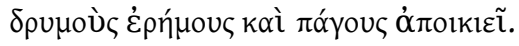

$$
\begin{aligned}
& \text { (Sofocle, fr. } 581 \text { Radt = } 14 \text { Milo) }
\end{aligned}
$$

L'upupa è un uccello straniero ${ }^{23}$, poco noto nell'Atene dell'età classica, caratterizzato da un aspetto strano, selvaggio e minaccioso, da un temperamento solitario e asociale ${ }^{24}$ - avendo esso abbandonato i suoi luoghi nativi - e abitudini sporche ${ }^{25}$. È un cacciatore 
di rondini $\mathrm{i}^{26}$, dominato da risentimento e da atteggiamenti ostili alle donne, tratti che devono essere ascritti alla sua vicenda umana. Il suo aspetto - che condensa tutto ciò che è ritenuto insolito ad Atene - è simile a quello di un uomo armato ${ }^{27}$ : la sua cresta

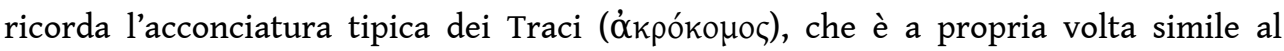
cimiero di un elmo ${ }^{28}$, mentre il suo becco lungo e appuntito ricorda una lancia ${ }^{29}$. Ciò lo

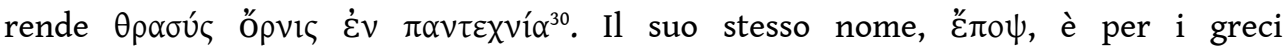
verosimilmente legato alle lingue barbariche ${ }^{31}$. L'upupa migra in Grecia, soggiornandovi in primavera e in autunno, ma non vi nidifica; lo fa, invece, in Macedonia e, forse, in Epiro ${ }^{32}$. Alla luce di queste considerazioni, la trasformazione finale di Tereo in un'upupa può essere interpretata come un ulteriore simbolo di barbarie. Ciò è evidente non solo nella perdita, da parte di Tereo, della natura umana attraverso l'imbestiamento ${ }^{33}$, ma anche nel fatto che la trasformazione in un uccello è un risultato che si addice al peggior tipo di reati contro la famiglia: gli uccelli sono lontani dalla specie umana più di quanto non siano altri animali, che presentano una maggior quantità di elementi comuni con gli uomini ${ }^{34}$.

Un ultimo elemento, che sembrerebbe provare l'ostilità di Sofocle nei confronti dei Traci, è osservabile nel seguente frammento:

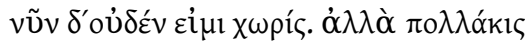

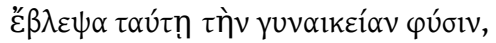

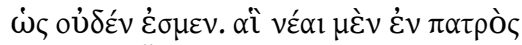

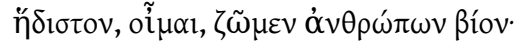

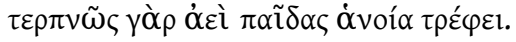

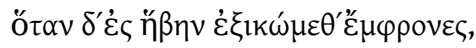

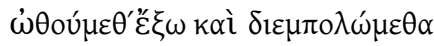

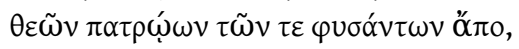

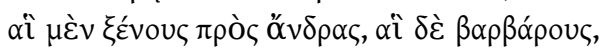

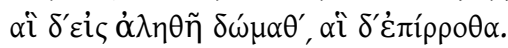

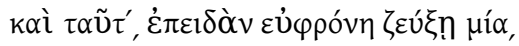

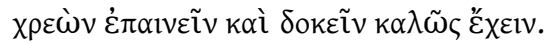

(Sofocle, fr. 583 Radt $=2$ Milo)

Qui, Procne si riferisce esplicitamente al proprio popolo con l'appellativo di «Greci», e successivamente deplora la sorte delle donne, vendute come merce dai propri padri a stranieri o barbari. È, dunque, osservabile una distinzione cosciente fra ל́́vol e $\beta \alpha \alpha \rho \beta \alpha \rho o l$. Se si analizza l'intera produzione di Sofocle, appare chiaro non solo che ebbe un profondo interesse per i popoli stranieri, ma anche che rappresentò molti di loro con la maggiore esattezza possibile nei drammi non conservati. Perciò, la differenziazione operata fra $\xi \dot{v} v o l$ e $\beta \alpha ́ p \beta \alpha \rho o l$ risulta essere estremamente significativa. Benché l'atteggiamento di Sofocle rispetto agli stranieri appaia caratterizzato da interesse e curiosità, l'antitesi fra greci e barbari è qui consapevolmente rappresentata, probabilmente per raffigurare l'alleanza militare coi Traci come dannosa ${ }^{35}$.

Le considerazioni sul Tereo, che ho appena esposto, possono essere integrate da un breve confronto con le Metamorfosi di Ovidio (VI, 420-675), che con ogni probabilità riferiscono il mito dell'usignolo rifacendosi a questo dramma. Nelle Metamorfosi (VI, 614-619), Procne esprime i suoi propositi di vendetta nei confronti di Tereo e, in questa sede, fornisce un elenco degli organi di cui vorrebbe mutilarlo. La lingua allude alla glossotomia subita da Filomela, così come gli occhi richiamano il nome di Tereo, colui che guarda, mentre i genitali si riferiscono alla sua insaziabile sessualità. Nella poesia di Ovidio è, dunque, osservabile una precisa rete simbolica, che si manifesta nella 
menzione della lingua, organo collegato all'elevata cultura greca, in contrasto con gli occhi, i quali rimandano direttamente a Tereo, e coi genitali, organi collegati ad un uso più rozzo e immediato, che ritengo alludano alla cultura barbarica ${ }^{36}$. Data la tematizzazione del contrasto fra civiltà e barbarie, evidente nel Tereo, è ragionevole ipotizzare che il menzionato elenco fosse già presente nel dramma di Sofocle. Verosimilmente, le procedure osservabili in questo passo delle Metamorfosi furono già realizzate, almeno in parte, da Sofocle, il quale - imitato da Ovidio - configurò l'opposizione fra le principesse ateniesi e Tereo come un'opposizione fra $\pi \alpha 1 \delta \varepsilon i ́ \alpha$ e barbarie.

Sofocle sembra, dunque, operare una rilettura del mito dell'usignolo che lo rifunzionalizza in chiave politica: essa ha il fine di comunicare l'avversione di Sofocle ad ogni possibile politica di alleanza con la Tracia ${ }^{37}$.

\subsection{Risemantizzazione dei nomi di Zeto e Tereo}

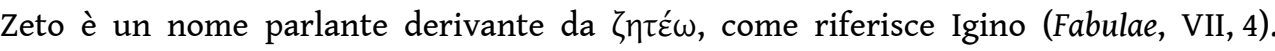
È, dunque, possibile interpretare il nome Zeto come cercatore; egli era, cioè, un uomo che cercava sua moglie, per punirla. Probabilmente, fu proprio in virtù di ciò, che Zeto fu scelto, nel mito, come sposo di Aedon. Non a caso, anche Tereo è un nome parlante, derivante da $\tau \eta \rho \varepsilon ́ \omega^{38}$. Il personaggio col nome di Tereo, nomen agentis che indica l'uomo che guarda, si identifica col proprio sguardo lascivo, che è la base delle sue azioni violente. Tale nome è, inoltre, associato a quello di Tere, che regnava sulla Tracia negli anni precedenti la rappresentazione del Tereo. Infine, è opportuno osservare che la parola ह̌ $\pi о \psi$, che indica l'upupa in cui Tereo si trasforma, ha lo stesso significato assunto dal nome Tereo ${ }^{39}$. La somiglianza dell'etimologia popolare di Tereo (da $\left.\tau \eta \rho \varepsilon ́ \omega\right)$ e

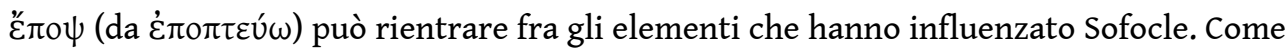
Tereo è l'uomo che guarda, l'upupa è, dunque, l'uccello che guarda. È inoltre possibile, a mio avviso, osservare una certa ironia tragica relativamente al nome parlante Tereo: Tereo avrebbe, effettivamente, dovuto guardare, sorvegliare Filomela, anziché stuprarla e mutilarla.

19 I nomi di Zeto e Tereo vengono entrambi rimotivati attraverso l'accostamento a due verbi per rafforzare la loro identità. Tale sorte apre nuove vie all'interpretazione del mito che - in due momenti storici differenti - vede entrambi protagonisti ${ }^{40}$.

\section{Influssi asiatici ed elementi metapoetici nei miti di Anfione e Zeto e delle loro spose}

\subsection{I rapporti del mito di Anfione e Zeto con Tantalo, Pandareo e il mito del Cane d'Oro}

20 Gli idionimi utilizzati nelle fonti della versione arcaica dei miti connessi ad Anfione, Zeto e alle loro spose si collegano a due ambienti diversi: quello microasiatico, di cui fa parte Pandareo (protagonista, insieme a Tantalo, del mito del Cane d'Oro ${ }^{41}$ ), e quello greco-continentale di cui fa parte Zeto ${ }^{42}$.

21 La presenza di Zeto nel mito di Aedon riconduce a Tebe. In questa tradizione è, dunque, osservabile l'accostamento di Zeto con Pandareo, proveniente dall'Asia. È possibile, 
inoltre, rilevare un parallelo fra la coppia Zeto-Aedon e la coppia Anfione-Niobe; quest'ultima è figlia di Tantalo e proviene a propria volta dall'Asia. Entrambi i fondatori di Tebe appaiono, dunque, già intimamente uniti con due eroi d'Asia, Tantalo e Pandareo, con i quali risultano acquisire lo stesso grado di parentela. A loro volta, Tantalo e Pandareo si trovano già associati nel mito del Cane d'Oro in una tradizione, che si colloca al di fuori della Grecia continentale. Non a caso, gli scoli ad Omero riferiscono una tradizione del mito, in cui, come motivazione della sconsideratezza di Aedon, viene introdotta Niobe; la sconsideratezza di Aedon viene spiegata con l'invidia di questa per la numerosa prole della cognata, esattamente come avveniva nel mito di Niobe $^{43}$. Niobe e Aedon restano entrambe prive di prole, piangendo in eterno la propria sconsideratezza, anche al di là della vita umana: esse sono, infatti, associate in una metamorfosi, che le priva della natura e della condizione di esseri umani ${ }^{44}$.

\subsection{Interazioni del mito di Niobe col mito di Aedon}

È possibile osservare che Niobe, nell'ambito della versione arcaica del mito di Aedon, viene descritta in modo molto diverso rispetto a quanto avviene nei versi dell'lliade che la riguardano strettamente (XXIV, 599-620). Nell'Iliade, tutti e dodici i figli di Niobe vengono uccisi da Apollo, che si propone così di punire la tracotanza della loro madre; la cognata di Niobe Aedon non prende in alcun modo parte alla strage. Riguardo a Niobe ed alla morte dei Niobidi, sono, dunque, probabilmente esistite due versioni, l'una indipendente dall'altra. La Niobe dell'Iliade, che fu trasformata in una roccia, appartiene verosimilmente ad una tradizione diversa da quella osservabile nel mito di Aedon. Ferecide, nel momento in cui riferisce specificamente la saga di Niobe, segue la versione dell'Iliade, in base a cui Niobe aveva sei figlie e sei figli, fra i quali non compare Amaleo ${ }^{45}$. Lo stesso Ferecide sembra, in questo modo, effettuare degli adattamenti programmatici rispetto a quanto aveva detto sul mito di Aedon, e sembra attingere a due saghe in origine diverse ${ }^{46}$.

23 Alla tradizione prettamente tebana di Anfione e Zeto si collegano Neide, - fratello di Itilio - Tebe, sposa di Zeto (invece di Aedon) ${ }^{47}$ e Ippomedusa, che era sposa di Anfione invece di Niobe ${ }^{48}$; essa era verosimilmente estranea all'influsso delle saghe asiatiche relative a Tantalo e Pandareo ${ }^{49}$. In un secondo tempo, a questa tradizione viene collegato il mito dell'usignolo, che giunge dall'Asia ad annunciare la primavera.

Questo nucleo subisce l'influsso della saga di Niobe. Aedon, che inizialmente era connotata solo come cognata di Niobe, assume, poi, il carattere di quest'ultima. Così Zeto subisce, per colpa della sposa asiatica, la stessa sorte di Anfione: la privazione della

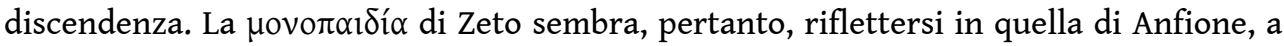
differenza di quanto avveniva nella saga di Niobe e Leto. Dunque, Amaleo non è uno dei Niobidi che muore prima della strage dei fratelli, in quanto la saga di Niobe è doppia. Le due spose di origine asiatica - discendenti da tracotanti sovrani asiatici - distruggono le stirpi degli ecisti di Tebe e, appaiono, dunque, meritare una pena che trascenda la loro stessa natura. La soppressione delle stirpi di Anfione e Zeto appare un destino estremamente grave per dei re; $i$ due fondatori di Tebe si trovano uniti in tale singolare destino, a causa delle loro spose di origine asiatica. La roccia e l'usignolo - in cui Niobe e Aedon rispettivamente si trasformano - allontanano le due eroine dalla natura umana, molto più di quanto non avvenga in altre metamorfosi note al pubblico greco ${ }^{50}$. 


\subsection{La lira di Anfione e il canto dell'usignolo come metafora della poesia lirica} canti di lavoro comuni in Grecia durante la costruzione di edifici ${ }^{51}$. Il mito di Anfione è, dunque, interpretabile come la resa poetica di questo costume e, dal momento che i toni della lira di Anfione costruirono le mura di Tebe, può essere letto alla luce dell'antico motivo del potere magico della musica e, conseguentemente, del mito ${ }^{52}$.

Allo stesso modo, osservando i riferimenti al mito dell'usignolo, è possibile notare come esso, che trae dal più grande dolore immaginabile - la morte del proprio figlio - il più bel canto esistente, assurga a simbolo del canto poetico. Non a caso, nell'Odissea (XIX, 518-524) viene usato, in relazione a questo mito, il verbo $\alpha \dot{\alpha} \delta \omega \omega$, che solitamente indica il solo cantare umano. In contesti tragici, l'usignolo ricorre quasi esclusivamente nelle sezioni liriche ${ }^{53}$ ed è generalmente utilizzato come termine di paragone per le sofferenze di chi lo invoca. Spesso il lamento del personaggio, che si paragona

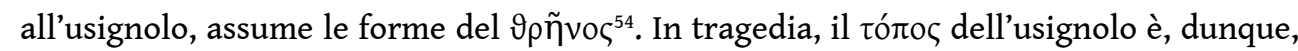
sempre invocato in relazione al canto tragico; non a caso, nelle Supplici (v. 65), in riferimento all'usignolo, Eschilo usa il verbo $\xi u v \tau i ́ \theta \eta \mu$, verbo della composizione letteraria ${ }^{55}$.

\section{BIBLIOGRAFIA}

ANDÒ Valeria, L'ape che tesse. Saperi femminili nella Grecia antica, Roma, Carocci, 2005.

BÄBLER Balbina, Niobe, in H. Cancik, H. Schneider et al. (edd.), Der neue Pauly. Enzyklopädie der Antike. Das klassische Altertum und seine Rezeptionsgeschichte, Stuttgart, Metzler, 2003.

BERMAN Daniel W., The Double Foundation of Boeotian Thebes, «TAPhA», 134, 2004, pp. 1-22.

BERMAN Daniel W., Myth Literature and the Creation of the Topography of Thebes, Cambridge, Cambridge University Press, 2015.

BIGA Anna M., L'Antiope di Euripide, Trento, Università degli studi di Trento, 2015.

BIRAUD Michèle \& DELBEY Evrard, Térée, Procné et Philomèle: du mythe aitiologique au début du mythe littéraire, in A. Zucker \& M. C. Olivi (edd.), Actes du $38^{e}$ Congrès international de l'APLAES, Nice, 2005, pp. 25-49.

BLOK Josine H., Gentrifying Genealogy: On the Genesis of the Athenian Autochthony Myth, in U. Dill \& C. Walde (edd.), Antike Mythen. Medien Transformationen und Konstruktionen, Berlin-New York, De Gruyter, 2009, pp. 251-275.

BURNETT Anne P., Revenge in Attic and Latter Tragedy, Berkeley, University of California Press, 1998. 
CALDER William M., Sophocles' Tereus: A Thracian Tragedy, in V. I. Gorciev, V. Täpkova-Zaimova \& V. Velkov (edd.), Thracia II (Academia Litterarum Bulgarica, Primus Congressus Studiorum Thracicorum), Sofia, Bulgarian Academy of Science, 1974, pp. 87-91.

CASANOVA Angelo, Osservazioni sui frammenti del Tereo, in G. Avezzù (ed.), Il dramma sofocleo: testo, lingua, interpretazione, Stuttgart-Weimar, Metzler, 2003, pp. 59-68.

CAZZANIGA Ignazio, Influsso della Medea di Euripide sul Tereo di Sofocle, «RIL», 69, 1935, pp. 433-438.

CAZZANIGA Ignazio, La saga di Itis nella tradizione letteraria e mitografica greco-romana I- Da Omero a Nonno Panopolitano, Milano-Varese, Istituto editoriale cisalpino, 1950a.

CAZZANIGA Ignazio, La saga di Itis nella tradizione letteraria e mitografica greco-romana II - L'episodio di Procne nel libro sesto delle Metamorfosi di Ovidio: ricerche intorno alla tecnica poetica ovidiana, Milano-Varese, Istituto editoriale cisalpino, 1950b.

DAVIES Malcolm, The Theban Epics, Washington, Centre for Hellenic Studies, 2015.

DOBROV Gregory, The Tragic and the Comic Tereus, «AJP», 114 (2), 1993, pp. 189-234.

FITZPATRICK David, Sophocles' Tereus, «CQ», 51, 2001, pp. 90-101.

FONTENROSE Joseph, The Sorrows of Ino and Procne, «TAPhA», 79, 1948, pp. 125-167.

GAMBARARA Daniele, Alle fonti della filosofia del linguaggio: lingua e nomi nella cultura greca arcaica, Roma, Bulzoni, 1984.

GERNET Louis, La légende de Procne et la date du Tereus de Sophocle, in L. Gailly (ed.), Mélanges offerts à O. Navarre par ses élèves et ses amis, «AC», 6 (2), 1935, pp. 207-217.

GROSS Walter H., Zethos, in A. Pauly, G. Wissowa, W. Kroll et al. (edd.), Paulys Realencyclopädie der classischen Altertumwissenschaft: neue Bearbaitung, Stuttgart, Metzler, 1894-1980.

HALL Edith, Inventing the Barbarian: Greek Self-Definition through Tragedy, Oxford, Oxford Clarendon Press, 1989.

HARMON Roger, Zethos, in H. Cancik, H. Schneider et al. (edd.), Der neue Pauly. Enzyklopädie der Antike. Das klassische Altertum und seine Rezeptionsgeschichte, Stuttgart, Metzler, 2003.

HOURMOUZIADES Nicolaos C., Sophocles' Tereus, in T. B. L. Webster, J. H. Betts, J. T. Hooker et al. (edd.), Studies in Honour of T. B. L. Webster, Bristol, Bristol Classical Press, 1986, pp. 134-142.

HURST André, Bâtir les murailles de Thèbes, in P. Bernardini (ed.), Presenza e funzione della città di Tebe nella cultura greca (Atti del Convegno internazionale, Urbino, 7-9 luglio 1997), Pisa-Roma, Istituti editoriali e poligrafici internazionali, 2000.

KISO Akiko, The Lost Sophocles, New York, Vanguard Press, 1984.

KRAPPE Alexander H., The Legend of Amphion, «CJ», 21 (1), 1925, pp. 21-28.

KÜHR Angela, Als Kadmos nach Boiotien kam. Polis und Ethnos im Spiegel thebanischer Gründungsmythen, Stuttgart, Steiner, 2006.

LESKY Albin, Niobe, in A. Pauly, G. Wissowa, W. Kroll et al. (edd.), Paulys Realencyclopädie der classischen Altertumwissenschaft: neue Bearbaitung, Stuttgart, Metzler, 1894-1980.

MAYER Maximilian, Mythistorica - I. Megarische Sagen, «Hermes», 27, 1892, pp. 487-489.

MILO Daniela (ed.), Il «Tereo» di Sofocle, Napoli, D’Auria, 2008.

MONELLA Paolo, Procne e Filomela: dal mito al simbolo letterario, Bologna, Pàtron, 2005. 
MÜHLL Peter VON DER (ed.), Homeri Odyssea, Basel, Helbing \& Lichtenhahn, 1962.

NEUDECKER Richard, Amphion, in H. Cancik, H. Schneider et al. (edd.), Der neue Pauly. Enzyklopädie der Antike. Das klassische Altertum und seine Rezeptionsgeschichte, Stuttgart, Metzler, 2003.

NEUHAUSEN Karl A., Tereus und die Danaiden bei Aischylos, «Hermes», 97 (2), 1969, pp. 167-186.

ODER Eugen, Der Wiedehopf in der griechischen Sage, «RhM», 43, 1888, pp. 541-556.

PEARSON Alfred C., The Fragments of Sophocles, Amsterdam, Hakkert, 1963.

RADKE Gerald, Prokne, in A. Pauly, G. Wissowa, W. Kroll et al. (edd.), Paulys Realencyclopädie der classischen Altertumwissenschaft: neue Bearbaitung, Stuttgart, Metzler, 1894-1980.

RADT Stefan (ed.), Tragicorum Graecorum Fragmenta, Vol. 4: Sophocles (Editio correctior et addendis aucta), Göttingen, Vandenhoeck \& Ruprecht, 1999.

RIBBECK Otto, Die römische Tragödie im Zeitalter der Republik, Leipzig, Teubner, 1875.

ROBERT Carl, Amphion, in A. Pauly, G. Wissowa, W. Kroll et al. (edd.), Paulys Realencyclopädie der classischen Altertumwissenschaft: neue Bearbaitung, Stuttgart, Metzler, 1894-1980.

ROSATI Giampiero \& CHIARINI Gioacchino (edd.), Ovidio: Metamorfosi. Volume III, libri V-VI, RomaMilano, Valla, 2013.

SCARPI Paolo, L'espace de la transgression et l'espace de l'ordre. Le trajet de la famille du mythe de Tereus au mythe de Kéléo, «DHA», 8, 1982, pp. 213-225.

SCATTOLIN Paolo, Le notizie sul Tereo di Sofocle nei papiri, in A. Casanova \& G. Bastianini (edd.), I papiri di Eschilo e di Sofocle (Atti del Convegno internazionale di studi, Firenze, 14-15 giugno 2012), Firenze, Firenze University Press, 2013, pp. 119-141.

SOMMERSTEIN Alan, FITZPATRICK David \& TALBOY Thomas (edd.), Sophocles: Selected Fragmentary Plays, Vol. 1: Hermione, Polyxene, The Diners, Tereus, Troilus, Phaedra, Oxford, Aris \& Phillips Classical Texts Oxford, 2006.

SUTTON Dana F., The Lost Sophocles, Lanham, New York, London, University Press of America, 1984.

THRÄMER Eduard, Aëdon, in A. Pauly, G. Wissowa, W. Kroll et al. (edd.), Paulys Realencyclopädie der classischen Altertumwissenschaft: neue Bearbaitung, Stuttgart, Metzler, 1894-1980.

THOMPSON D’Arcy W., A Glossary of Greek Birds, Hildesheim, Olms, 1966.

TSITSIBAKOU-VASALOS Evanthia, Ancient Poetic Etymology. The Pelopids: Fathers and Sons, Stuttgart, Steiner, 2007.

TULLI Mauro, Il Gorgia e la lira di Anfione, in M. Erler \& L. Brisson (edd.), Gorgias-Menon; selected papers from the Seventh Symposium Platonicum (proceedings), Sankt Augustin, Akademia, 2007, pp. 72-77.

WALDNER Katharina, Prokne, in H. Cancik, H. Schneider et al. (edd.), Der neue Pauly. Enzyklopädie der Antike. Das klassische Altertum und seine Rezeptionsgeschichte, Stuttgart, Metzler, 2003.

WELCKER Friedrich G., Die griechischen Tragödien mit Rücksicht auf den epischen Cyclus, Bonn, Weber, 1839.

ZACHARIA Katerina, "The Rock of the Nightingale": Sophocles' Tereus and Kinship Diplomacy, in F. \&

P. Michelakis (edd.), Homer, Tragedy and Beyond: Essays in Honour of P. E. Easterling, London, Society for the Promotion of Hellenic Studies, 2001. 


\section{NOTE}

1. Il nome di Cadmo era, inoltre, legato alla civilizzazione: Cadmo era considerato il portatore delle lettere in Grecia, in quanto si riteneva che l'alfabeto fosse nato in Beozia; vedi anche Kühr (2006, 87-106).

2. Vedi anche Berman (2004, 2-3).

3. Secondo Asio, sono figli di Epopeo di Sicione (Asio, fr. 1 West).

4. Secondo [Apollodoro], Biblioteca, III, 10, 1, sposò Tebe.

5. Vedi anche Euripide, Fenicie, 145; Pausania, IX, 5, 9.

6. Vedi anche Euripide, Ecuba, 29.

7. Si tratterebbe di una figlia femmina secondo una fonte non ferecidea di: Scholium in Euripidis, Phoenissas, 1111, p. I, 364 Schwartz.

8. Vedi anche Scholium in Apollonium Rhodium, IV, 1090, p. 305 Wendel; Properzio, III, 15, 29-42; Igino, Fabulae, VIII. Estremamente significativi risultano essere i frammenti 184-186 e 188 Kannicht dell'Antiope, che riportano parte dell'agone fra Anfione e Zeto. L'agone ha per tema la scelta fra la vita d'azione, rappresentata paradigmaticamente da Omero e incarnata - nel caso specifico - da Zeto, e la vita contemplativa, simboleggiata dalla lira di Anfione; vedi anche Tulli (2007, 74), Biga (2015, 217-262).

9. Vedi anche Tulli (2007, 72-77).

10. Il loro numero varia, nelle fonti, fra tre e venti. Omero (Iliade, XXIV, 614-617) parla di sei figli maschi e sei femmine.

11. Esiodo, fr. 183 M.-W.; Ferecide, III, fr. 38 Jacoby; Saffo, fr. 142 Voigt; Eschilo, fr. 154a-163 Radt; Sofocle, fr. 441a-451 Radt.

12. Ferecide, III, fr. 124 Jacoby; la versione di Ferecide è desumibile dagli Scholia in Odysseam, XIX, 518, p. II, 683 Dindorf e da Eustazio in Odysseam, XIX, $518(1875,32)$.

13. Scholium in Odysseam, XIX, 518 B2, p. II, 683 Dindorf. È opportuno, in ogni caso, osservare che lo scolio $\mathrm{B} 2$ è molto probabilmente l'esito di successivi rimaneggiamenti; in virtù di ciò, non potrebbe essere propriamente annoverato fra le fonti della versione arcaica del mito.

14. Eschilo, Supplici, 57-67, Agamennone, 1140-1149; Sofocle, Aiace, 625-634, Trachinie, 103-111, 962-963, Elettra, 103-109, 145-152, 1074-1081, Edipo a Colono, 668-678; Euripide, Andromaca, 861-862, Eracle, 1016-1027, Elena, 1107-1116, Fenicie, 1509-1522, Fetonte, 67-70.

15. Mi riferisco alla versione del mito riportata da Sofocle, il quale, nel Tereo, sviluppò tale mito nella sua forma più completa; gli altri autori si limitano a farvi dei brevi cenni.

16. Tucidide, II, 29,3; vedi anche Pausania, I, 41, 8-9; IV, 8-9.

17. Di questo avviso sono: Mayer (1892, 491), Cazzaniga (1935, 438; 1950a, 61-63), Gernet (1935, 209-216), Kiso (1984, 60), Dobrov (1993, 213), Monella (2005, 87-88).

18. Ciò che Filomela ricama è molto probabilmente un messaggio scritto, com'è osservabile dalle seguenti testimonianze: Scholium in Aristophanis, Aves, 212, pp.37-38 Holwerda; Aristotele, Poetica, 1454 b 35-37; Ovidio, Metamorfosi, VI, 577, 582; [Apollodoro], Biblioteca, III, 14, 8; Libanio, Narrationes, XVIII, 2. Di questo avviso sono Calder $(1974,89)$, Kiso $(1984,67)$, Dobrov $(1993$, 204-205). Di un altro avviso sono, invece, Ribbeck $(1875,580)$, il quale ha ipotizzato una compresenza di lettere e disegni, e Pearson $(1963,221)$ che, seguito da Casanova $(2003,59-68)$, si è espresso a favore di un'immagine ricamata. Hourmouziades $(1986,137)$ appare indeciso fra lettere e disegni. Monella $(2005,109)$ si è limitato a constatare che la maggior parte degli studiosi ha ipotizzato l'uso di un messaggio scritto. Pur ritenendo opportuno mantenere una certa

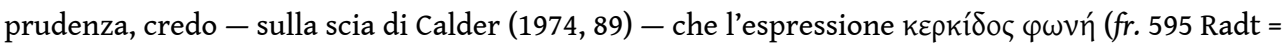
7 Milo) alluda con ogni verosimiglianza ad un messaggio scritto, dal momento che le iscrizioni greche venivano normalmente lette ad alta voce.

19. Sofocle, fr. 595 Radt (7 Milo); vedi anche Dobrov (1993, 202), Monella $(2005,106)$. Scattolin $(2013,126)$ osserva che lo stratagemma della «voce della spola» è innovazione sofoclea: Aristotele 
(Poetica, 1454 b 35-37) non avrebbe avuto alcun interesse a citare, in relazione al Tereo, un elemento attestato anche nelle versioni precedenti del mito.

20. Vedi anche Burnett (1998, 186-187). Nel Tereo, Procne riesce a liberare Filomela in occasione delle Trieteriche, feste tracie in onore di Dioniso, verosimilmente ricorrendo a dei travestimenti da menadi. Tale stratagemma alluderebbe al fatto che, grazie alla cultura ateniese, gli elementi tipici delle civiltà barbariche si rivoltino contro i barbari stessi.

21. Platone, Simposio, 205 b.

22. Erodoto, II, 82.

23. Pausania, I, 41, 8-9.

24. Sofocle, fr. 581, 9-10 Radt (14 Milo).

25. Si riteneva che realizzasse il proprio nido con escrementi umani (Aristotele, Historia Animalium, 616 a 35).

26. Come riferisce Eliano (Storie Varie, II, 3). In realtà, l'upupa è un uccello insettivoro molto timido e pauroso, che teme perfino le rondini; vedi anche Oder $(1888,546)$.

27. Così lo descrivono Sofocle (fr. 581, 3 Radt = 14 Milo) e Ovidio (Metamorfosi, VI, 674).

28. Omero, Iliade, IV, 533; vedi anche Thompson $(1966,96)$.

29. Vedi Ovidio, Metamorfosi, VI, 673-674.

30. Sofocle, fr. 583, 1 Radt (2 Milo). Probabilmente, Sofocle rappresentò Tereo vestito da upupa sulla scena; da qui l'accusa mossa dalla controparte comica di Tereo a Sofocle, indicato come responsabile del suo aspetto ridicolo (Aristofane, Uccelli, 98-102).

31. Aristofane, Uccelli, 199-201; vedi anche Scarpi (1982, 213-217).

32. Aristotele, Historia Animalium, 559 a 8; vedi anche Thompson (1966, 96).

33. Vedi anche Kiso (1984, 81), Monella (2005, 124-125).

34. Vedi anche Zacharia $(2001,96)$.

35. Fra i tre tragici, Sofocle fu sempre considerato quello meno interessato agli stranieri, poiché, nelle sue opere conservate, rappresentò un minor numero di elementi riconducibili a popoli stranieri rispetto ad Eschilo ed Euripide. Inoltre, a Sofocle fu legata l'immagine tradizionale di poeta classico e puramente greco, la quale portò a credere che non fosse interessato al mondo esterno ad Atene. Ma, se si analizzano i suoi drammi frammentari, appare chiaro che avesse un profondo interesse per i popoli stranieri (Andromeda, Tamira, Colchidi, Rhizotomoi, Sciti, Laocoonte, Alessandro, Antenoridi, Troilo, Trittolemo, Enomao); vedi anche Kiso (1984, 51-53).

36. Vedi anche Rosati, Chiarini $(2013,344)$. Sutton $(1984,129)$ paragona lo stesso passo delle Metamorfosi al frammento 587 Radt (4 Milo) del Tereo. Entrambi i passi sembrano caratterizzati da pregiudizi sui barbari.

37. Interpretazioni diverse dalla mia sono offerte da Cazzaniga (1950a, 35-36), Dobrov (1993, 184), Casanova (2003, 59-68).

38. Scholium in Aristophanis, Aves, 102, pp. 21-22 Holwerda; vedi anche Neuhausen (1962, 172-173).

39. Vedi anche Fontenrose $(1948,155)$.

40. Nella poesia arcaica è, spesso, possibile osservare mutazioni implicite del carico etimologico e semantico di un nome. Tale risemantizzazione, spesso effettuata in modo allusivo e tuttavia comprensibile al pubblico, è funzionale alla creazione di una corrispondenza fra il nome del personaggio e il contesto poetico in cui questo si trova inserito; vedi anche Tsitsibakou-Vasalos $(2007,69)$. Sulle etimologie arcaiche vedi anche Gambarara $(1984,108-135)$.

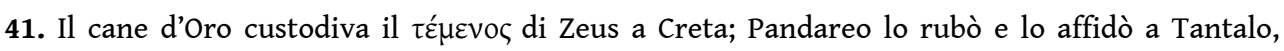
perché lo nascondesse a Zeus. Entrambi gli eroi furono, poi, soggetti, alla punizione del dio: Scholium in Odysseam, XIX, 518-520, p. II, 683 Dindorf; Scholium in Odysseam, XX, 66-68, p. II, 688 Dindorf; Eustazio in Odysseam, XIX, $518(1875,32)$.

42. Vedi Monella $(2005,18)$.

43. Scholia in Odysseam, XIX, 518-520, p. II, 683 Dindorf. 
44. Vedi anche Cazzaniga (1950a, 6-7).

45. Scholium in Euripidis, Phoenissas, 1111, p. I, 364 Schwartz.

46. Vedi Cazzaniga (1951a, 10).

47. [Apollodoro], Biblioteca, III, 10, 1.

48. [Apollodoro], Biblioteca, I, 29, 4 .

49. Vedi Cazzaniga (1950a, 11).

50. Vedi Cazzaniga (1950a, 7, 12).

51. Pausania, IX, 5, 6.

52. Vedi Krappe $(1925,28)$.

53. Fa eccezione Euripide, Eracle, 1039-1041.

54. Eschilo, Agamennone, 1141-1144; Sofocle, Aiace, 628-631, Trachinie, 964, Elettra, 104, 1076;

Euripide, Eracle, 1039, Elena, 1111-1115, Fenicie, 1515-1518.

55. Vedi anche Monella (2005, 221-233).

\section{RIASSUNTI}

L'obiettivo di questo contributo è di chiarire la ricezione del mito tebano di Zeto e della sua sposa Aedon in epoca classica attraverso un'analisi dei suoi adattamenti e interpretazioni. Lo spostamento dello sfondo del mito in Tracia può essere letto come una critica nei confronti di questa regione, con la quale Atene aveva in quegli anni dei rapporti difficili. Alcuni elementi deducibili dal Tereo, come la «voce della spola», monstrano che Sofocle ha proposto una rilettura del mito dell'usignolo in termini politici: in questo modo egli intese esprimere la sua avversità a proposito di una possibile alleanza ateniese con la Tracia. Si tratta di un problema capitale nel dibattito politico ateniese all'epoca del regno tracio di Tere e di Sitalce.

L'objectif de cette contribution est de clarifier la réception à l'époque classique du mythe du cycle thébain de Zéthos et son épouse Aédon à travers une analyse de ses adaptations et interprétations. Le déplacement du décor du mythe en Thrace peut être lu comme une critique envers cette région, avec laquelle Athènes avait des rapports difficiles à l'époque. Il y a des éléments déductibles du Térée, comme la "voix de la navette ", qui montrent que Sophocle a proposé une relecture du mythe du rossignol en termes politiques: par ce moyen, il veut exprimer son aversion contre la possibilité d'une alliance athénienne avec la Thrace. Il s'agit d'un problème capital dans le débat politique athénien au moment du gouvernement de la Thrace par Térès et Sitalcès.

By analyzing its adaptations and interpretations, this paper will shed light on the reception, in the classical period, of the Theban cycle myth of Zethus and his bride Aëdon. The myth's displacement resulting from its setting in Thrace can be read as a critique of Thrace itself, with which Athens had difficult relations with at the time. Some topics recognisable from Tereus, such as the "voice of the shuttle", show that Sophocles re-read the myth of the nightingale in political terms. The re-reading expresses Sophocles' aversion to any possible alliance with Thrace, a central issue in the Athenian political debate at a time when Thrace was under Teres' and Sitalces' government. 
INDICE

Mots-clés : Amphion, Zéthos, Aédon, Thèbes, tragédie, Térée

Keywords : Amphion, Zethus, Aedon, Thebes, tragedy, Tereus

Parole chiave : Anfione, Zeto, Aedon, Tebe, tragedia, Tereo

\section{AUTORE}

\section{SABRINA MANCUSO}

Eberhard Karls Universität Tübingen.

Sabrina Mancuso a obtenu son diplôme magistral en lettres classiques à l'université de Pise en 2015 avec un mémoire intitulé Un paradigma mitico: Issione nel Filottete di Sofocle. Depuis 2016, elle est inscrite en doctorat (cotutelle Eberhard Karls Universität Tübingen-Università di Pisa) et prépare une thèse intitulée Der Prokne-Mythos als exemplarischer Mythos bei Aischylos, Sophokles und Euripides. Ses principaux domaines de recherche sont la poésie homérique et son interprétation, la poésie épique archaïque et la tragédie, notamment les drames de Sophocle. 